\title{
Effect of Magnetostriction on Localised Flux Density in Toroidal Amorphous Cores Due To Bending Stress
}

\author{
Ismet Gucuyener \\ Vocation School of Technical Science, Uludag University, Gorukle Bursa, 16059, Turkey \\ E-mail: ismetguc@uludag.edu.tr \\ Naim Derebasi (Corresponding author) \\ Physics Department, Uludag University, Gorukle Bursa, 16059, Turkey \\ E-mail: naim@uludag.edu.tr
}

\begin{abstract}
Application of a force causes a localized bending stress, which results in a change in the domain structure and therefore a variation in induced voltages are occurred across search coils uniformly wound on this area. The magnetostriction of amorphous ribbons vary with their compositions. Two types of amorphous ribbon were used to analyse the variation of localised flux density with different flux density and frequency levels. Amorphous toroidal transducers were made from high and near zero magnetostrictive Metglas ${ }^{\circledR}$ as-cast amorphous ribbons, which were Fe-based 2605SA1 and Co-based 2705X respectively. Measurements were carried out to confirm the influence of applying force and frequency on output characteristic of the transducer due to their magnetostriction. A force was applied to the toroidal core in steps of 20 from 0 to $500 \mathrm{~g}$-force. The transducer under the test were sinusoidally magnetised over the frequency range from 1 to $10 \mathrm{kHz}$ and flux density levels of 50, 100 and $150 \mathrm{mT}$. The variation in the induced voltage of search coils located at $0^{\circ}$ and $90^{\circ}$ was very significant with applied stress, while it was less pronounced located at $45^{\circ}$ with applied stress on the Fe-based toroidal core and the applying force is not effective on $2705 \mathrm{X}$ amorphous ribbon. This investigation highlights the influence of localised bending stress on flux density in amorphous ribbon cores due to its magnetostriction.
\end{abstract}

Key words: Amorphous ribbon core, magnetostriction, flux distribution.

DOI: $10.7176 / J S T R / 6-07-13$

\section{Introduction}

Great elastic properties of amorphous ribbon materials combined with their excellent magnetic properties make them suitable for transducer and sensor applications. When the material is subjected to both magnetisation and applied stress, the resultant magnetic domain pattern is determined by the anisotropy energy [1]. A basic amorphous ribbon transducer is magnetised by an ac current applied to magnetising windings and then the search coils output voltage is a function of the applied force, induction frequency and bulk flux density. In amorphous ribbon core toroidal transducers, application of a force causes a localised varied bending stress, which results a change in the domain structure and therefore, a variation in the induced voltage of the search coils wound on this area. A tensile stress would be induced on the outer surface of the toroidal core while a compressive stress produced on the inner surface due to bending stress. Flux orientations due to the domain structure of ribbon under applied force were investigated by orthogonal search coils on the bent region [2].

This research is focused on the localised flux density variation detected on the search coil located at $0^{\circ}$, $45^{\circ}$ and $90^{\circ}$ of toroidal core transducers made from Fe and Co based amorphous ribbon Metglas ${ }^{\circledR}$ 2605SA1 and 2705X under applying force. Furthermore, the localised flux density variation was also investigated the materials, which have high or near zero magnetostriction.

\section{Experiment}

The transducers were constructed from 2605SA1 and 2705X amorphous ribbon material to form a 10wrap toroid. Both materials are commercially produced by Allied Signal Inc $^{\odot}$. with a chemical composition of $\mathrm{Fe}_{81.7} \mathrm{~B}_{16} \mathrm{Si}_{2} \mathrm{C}_{0.3}$ and $\mathrm{Fe}_{5.85} \mathrm{Co}_{70.15} \mathrm{Mo}_{4} \mathrm{~B}_{15} \mathrm{Si}_{5}$ respectively. Although, the 2605SA1 has high saturation magnetostriction, $\lambda_{S}=27 \times 10^{-6}$, the $2705 \mathrm{X}$ possesses nearly zero magnetostriction, $\lambda_{S}<1$. The transducers were made from these materials, which were $25.4 \mathrm{~mm}$ wide with a $30 \mathrm{~mm}$ inner diameter and they were 30 and $50 \mu \mathrm{m}$ thick respectively. An adhesive tape were gently covered on the edge of cores to protect the coil wires from being cut by the sharp edges of the ribbon and for short circuit. 
The toroidal transducers was magnetised by a primary winding with uniformly wound 100 turns and 0.1 $\mathrm{mm}$ search coil wire. The search coils were located on the transducers with different angles which were $0^{\circ}, 45^{\circ}$ and $90^{\circ}$ beginning from the contact point of transducer to the measuring apparatus [Fig. 1]. The components of flux vector may be detected on these search coils due to applying force and location angle during magnetisation. The search coils were uniformly wound identical to each other to minimise dimensional errors and influence the flux density calculations. The transducers made were mounted onto a force application apparatus as shown in Fig. 1. A force using basic weights was then applied to the toroid in steps of 20 from 0 to $500 \mathrm{~g}$-force.

The transducer under the test were sinusoidally magnetised by a waveform generator type Agilent HP33250A with the frequency range from 1 to $10 \mathrm{kHz}$, in the flux density levels of 50, 100 and $150 \mathrm{mT}$. The search coil voltages were read from a digital multimeter type Agilent HP3458A to analyse the variation of the flux density synchronised with applied force.

Peak magnetic flux density from the magnetising and search coils was determined by the following equation;

$$
B_{m}=\frac{V_{r m s}}{4.44 N A f}
$$

where $\mathrm{N}$ is the number of turns of the search coil, $\mathrm{A}$ is the cross sectional area (product of the width of search coils and thickness of transducers) and $\mathrm{f}$ is the magnetization frequency.

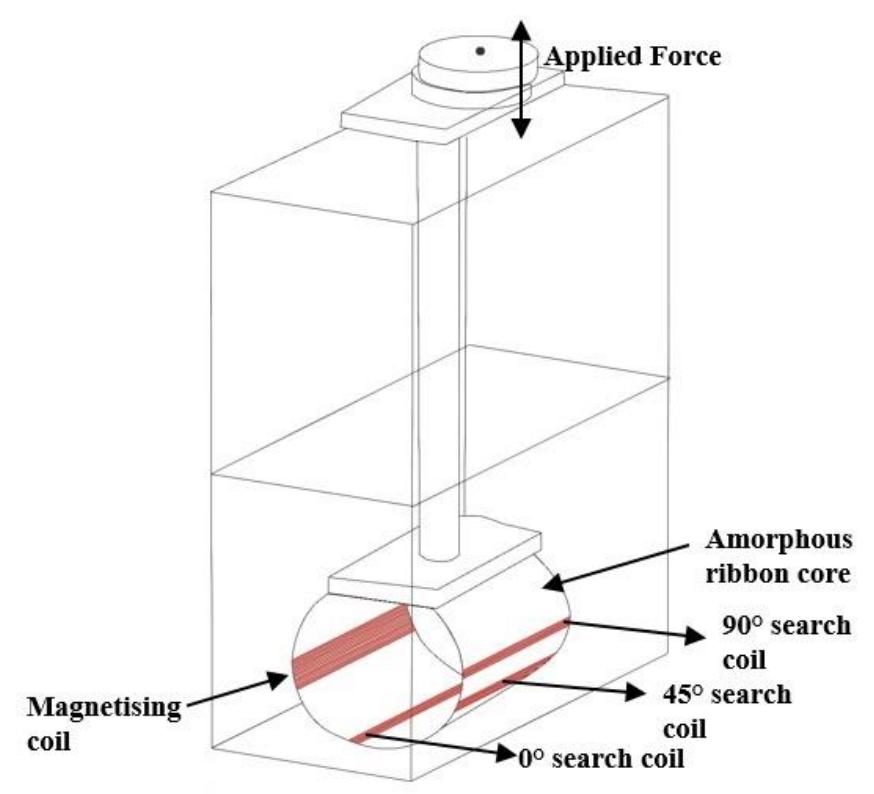

Fig. 1. A symbolic view of the force application apparatus.

\section{Results and Discussions}

When a toroidal core made of amorphous ribbon is exposed to a force, a stress occurs in the bent regions of the core. A tensile stress is induced on the outer surface of toroidal core and a compressive stress on the inner surface [1]. The application of a force to the transducer core will cause domain walls to move such as bar shape domains from parallel to the longitudinal direction on the outer surface of the bent region and perpendicular to this direction on the inner surface in a positive magnetostrictive materials. This behaviour can be understood in terms of the preferred domain orientation set up by applied stress. Stress alone can cause domain wall motion. This motion may be ensured zero net magnetisation for the whole sample. Stress can also be create an easy direction of magnetisation. Therefore, when stress is existed, stress anisotropy must be considered along with any other anisotropies that may exist. Magnetic elastic energy can be written for the stress anisotropy,

$$
E_{\lambda}=\frac{3}{2} \lambda_{S} \sigma \sin ^{2} \theta
$$


where $\lambda_{\mathrm{S}}$ is the saturation magnetostriction, $\sigma$ is the stress and, $\theta$ is the angle between the stress and saturation magnetisation, $M_{S}[3]$. When a material is magnetised, the magnetostriction changes with magnetisation as,

$$
\lambda=\frac{3}{2} \lambda_{S}\left(\frac{M}{M_{S}}\right)
$$

where $\lambda$ is the magnetostriction at the magnetisation $\mathrm{M}$.

Fig. 2 shows variation of localized coil output voltage with applied force on toroidal core 2605SA1 at bulk flux density range from 50 to $150 \mathrm{mT}$ and frequency from 1 to $10 \mathrm{kHz}$ respectively. Frequency and location angle are very significant on the localised induced voltage. The localised induced voltage detected from the search coils located at different angles was minimum at $1 \mathrm{kHz}$, even the bulk flux density was increased. However, the induced localised flux density was increased about $500 \%$ at $5 \mathrm{kHz}$ and the search coil located $90^{\circ}$ and bulk flux density level $150 \mathrm{mT}$. It is also increased about $173 \%$ at the same search coil and $10 \mathrm{kHz}$. The induced voltage was significantly varied at $150 \mathrm{mT}$ for the induced voltages of all search coils. A maximum change was detected on the search coil located angle at $90^{\circ}$ and then $0^{\circ}$ finally $45^{\circ}$. This behaviour can be explained by the role of domain structure in the bent region.
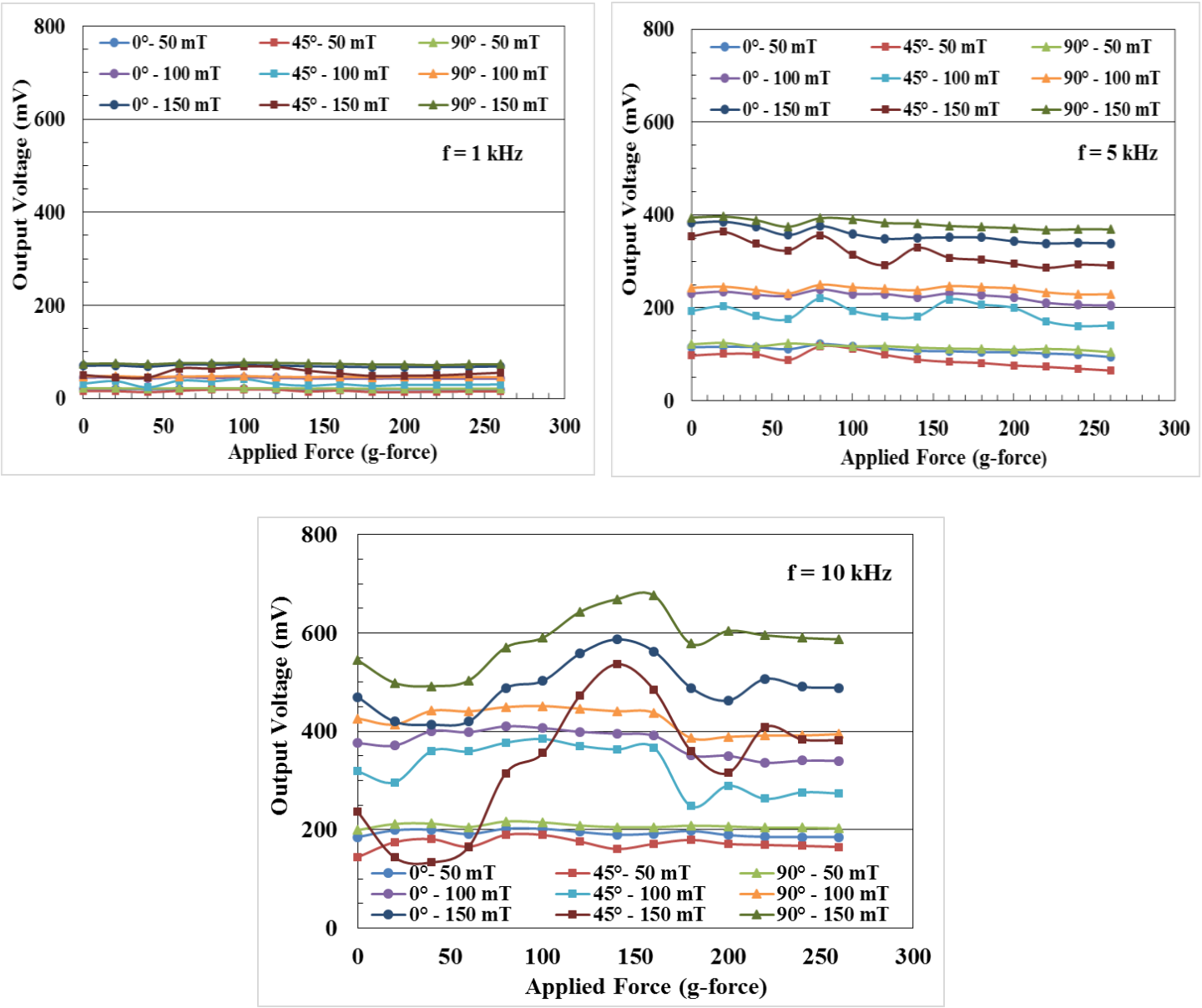

Fig. 2. Variation of localized coil output voltages with applied force on toroidal core 2605SA1 at 1 $\mathrm{kHz}, 5 \mathrm{kHz}$ and $10 \mathrm{kHz}$ respectively.

A high magnetostrictive amorphous ribbon such as 2605AS1 has certain bar domain structure without any applied force [2]. This domain structure on the bent region due to applied force can be formed such that the bar shape domains along the longitudinal direction on the outer surface are getting narrower while the domains on the inner surface on a different toroidal layer gradually changes the orientation 
along the transverse direction. This change is quite severe under the bent region of the search coils located angle at $0^{\circ}$ and $90^{\circ}$ as compared with $45^{\circ}$. Although, the bar domain structure along the longitudinal direction, the flux can get through onto the outer surface. However, some flux follows the transvers direction at the inner surface due to domain structure in this region. This behaviour is also indicated the importance of the stress induced anisotropy. The change in the localised induced voltage via localised flux and domain wall movement can cause the permeability change in the stress region. Thus, the core path reluctance increases.

The data obtained from toroidal core $2705 \mathrm{X}$ was indicated in Fig. 3. Whereas the induced voltage is more stable with applying force, it is increasing with the bulk flux density and frequency level. The toroidal core $2705 \mathrm{X}$ has zero or near zero magnetostriction and this ribbon is not efficiently used for high frequency applications. The stability of induced voltages detected from the search coils due to applying force can be explained by the high frequency characteristics of this ribbon. The domain structure is not significantly changed by applying force on $2705 \mathrm{X}$ due to the magnetostriction of the material and hence the induced voltage is almost kept constant at all flux density and frequency levels. High output voltage was obtained from the toroidal core $2705 \mathrm{X}$ due to high bulk flux density, similar to $2605 \mathrm{SA} 1$ at $10 \mathrm{kHz}$.
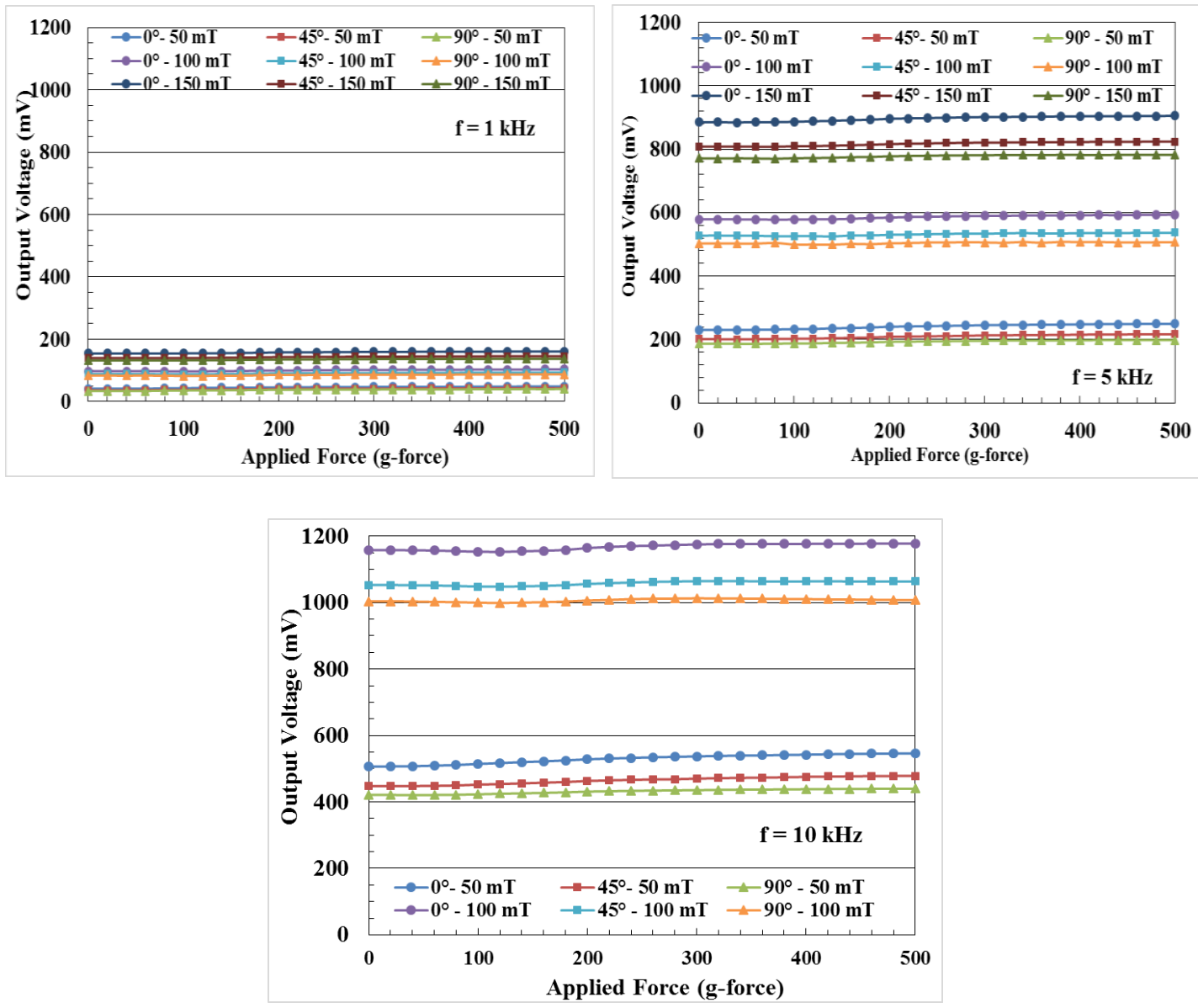

Fig. 3. Variation of localized coil output voltages with applied force on toroidal core $2705 \mathrm{X}$ at $1 \mathrm{kHz}, 5$ $\mathrm{kHz}$ and $10 \mathrm{kHz}$ respectively.

\section{Conclusions}

The domain wall movement due to applied force on the amorphous toroidal transducers is responsible for the induced voltage or localised flux density variations. This effect is more significant in high magnetostrictive amorphous materials. A severe change on localised flux density was detected on the search coils located at $0^{\circ}$ and $90^{\circ}$. A better working condition was obtained on the toroidal core 2605SA1 at $10 \mathrm{kHz}$ and $150 \mathrm{mT}$ combined with induced voltage, induced stress anisotropy and permeability or reluctance change in the bent region. This investigation highlights the influence of localised bending stress in highly magnetostrictive and near zero magnetostrictive amorphous ribbons. 


\section{References}

[1] T. Meydan, M. Goktepe, A. Honda, N. Derebasi, "Influence of bending stress on domain motion in amorphous material based magneto elastic transducers", J. Magn. Magn. Mater., vol. 112, pp. 269-271, 1992.

[2] N. Derebasi, T. Meydan, "Effect of stress on localised flux directions in amorphous toroidal transducers", Sensors and Actuators A, vol. 59, pp. 342-346, 1997.

[3] S. Chikazumi, "Physics of magnetism", Robert E. Krieger Publishing Co. Inc., Chapter 13, pp.260, 1986. 